\title{
Can 9am serum cortisol levels be used \\ to predict patient's response to the insulin tolerance test?
}

The Leeds Teaching Hospitals NHS

\section{N Kyriakakis, E Appleton, J Andrew, RD Murray}

Leeds Centre for Diabetes \& Endocrinology

\section{Introduction/Aim}

The insulin tolerance test (ITT) is a dynamic test to assess the $\mathrm{ACTH} / \mathrm{cortisol}$ and growth hormone (GH) reserve in patients with hypothalamo-pituitary disease. Contraindications for the test include history of epilepsy, ischaemic heart disease and age $>65$ years. The aim of this study is to evaluate if the 09.00am (pre-test) cortisol levels can be predictive of the patient's response to hypoglycaemia, minimizing the use of the ITT as it is a labour, intensive investigation and unpleasant for the patient.

\section{Methods}

This is a retrospective study of 110 ITTs performed at the Endocrine Unit of St James's University Hospital, Leeds, between January 2010 and July 2013. The test was performed on patients (age range 16-66) with a previous history of cranial surgery and/or radiotherapy for a variety of intracranial tumours, using the Trust's ITT protocol. For the results to be interpreted, hypoglycaemia of less than $2.2 \mathrm{mmol} / \mathrm{l}$ had to be achieved. Blood samples for cortisol and glucose levels were taken at 0 minutes (basal cortisol) and at $30,45,60,90$ and 120 minutes following insulin administration. A peak cortisol level:

$\bullet \geq 500 \mathrm{nmol} / \mathrm{l}$ was considered as a normal response

- 400-499 nmol/I was considered a suboptimal response

$-<400 \mathrm{nmol} / \mathrm{l}$ was considered as a failed response

\section{Results}

Table 1 shows a breakdown of the patients' response to hypoglycaemia in association with their baseline cortisol levels.

\begin{tabular}{|l|l|l|l|}
\hline $\begin{array}{l}\text { Baseline } \\
\text { cortisol } \\
\text { (nmol/I) }\end{array}$ & $\begin{array}{l}\text { Posititve ITT } \\
\text { (peak cortisol } \\
\geq 500 \mathrm{nmol} / \mathrm{I})\end{array}$ & $\begin{array}{l}\text { Suboptimal } \\
\text { response (peak } \\
\text { cortisol 400-499) }\end{array}$ & $\begin{array}{l}\text { Negative ITT } \\
\text { (peak cortisol } \\
<400 \mathrm{nmol} / \mathrm{I})\end{array}$ \\
\hline$<50$ & ---- & ---- & 4 \\
\hline $50-99$ & ---- & ---- & 2 \\
\hline $100-149$ & 1 & ---- & 2 \\
\hline $150-199$ & 6 & 9 & 3 \\
\hline $200-249$ & 9 & 6 & 8 \\
\hline $250-299$ & 6 & 3 & 4 \\
\hline $300-349$ & 13 & 6 & --- \\
\hline $350-399$ & 6 & 3 & 1 \\
\hline $400-449$ & 5 & 4 & --- \\
\hline $450-499$ & 3 & ---- & --- \\
\hline$\geq 500$ & 5 & 1 & --- \\
\hline Notal & & & \\
\hline
\end{tabular}

Table 1: Patients' response to hypoglycaemia in association with their baseline cortisol levels
Results (continued)

- Six patients had basal cortisol $<100 \mathrm{nmol} / \mathrm{l}$. All of them (100\%) failed to respond to hypoglycaemia.

- 57 had basal cortisol between 100-299nmol/l. 22 (38.6\%) of them had a normal ITT, 18 (31.6\%) had a suboptimal response and 17 (29.8\%) had an inadequate response.

- The remaining 47 patients had baseline cortisol $\geq 300 \mathrm{nmol} / \mathrm{I}$. 32 of them (68.1\%) responded normally, 14 (29.8\%) had a suboptimal response and 1 (2.1\%) failed the ITT.

Figure 1. summarizes the above results

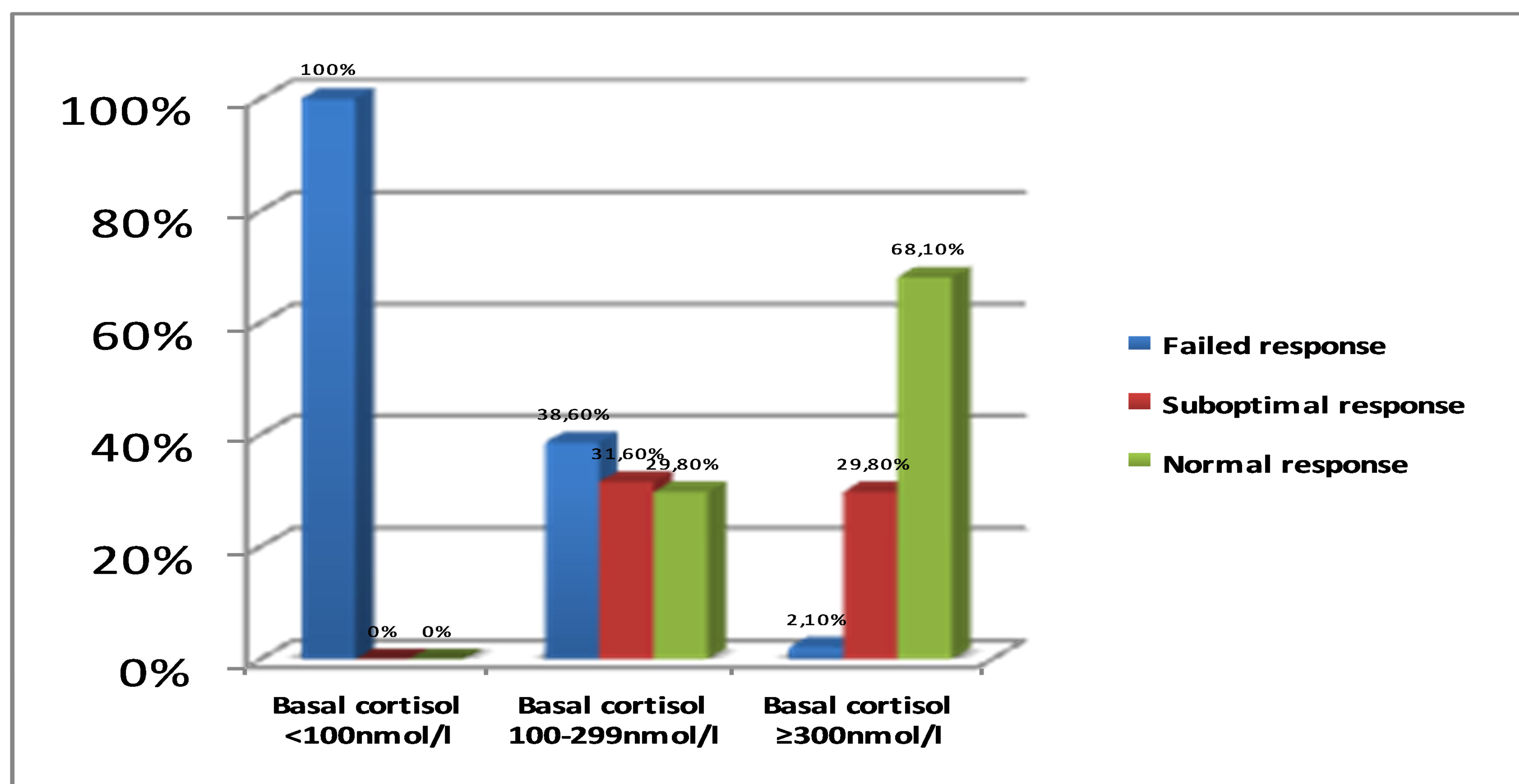

Figure 1: ITT outcomes according to baseline cortisol

\section{Conclusions}

Measurements of basal serum cortisol can identify patients for whom the ITT may not be necessary. Basal cortisol <100nmol/I indicated HPA axis insufficiency, whereas levels $\geq 300 \mathrm{nmol} /$ l suggest an at least suboptimal response to hypoglycaemia. In both groups the ITT could be avoided, as it would not alter patients' management. In our study $49.2 \%$ fall into this category. On the contrary, baseline cortisol between $100-299 \mathrm{nmol} / \mathrm{I}$ (51.8\% of our patients) cannot be used to predict patient's response to hypoglycaemia, therefore dynamic tests for the HPA axis assessment should be performed. Figure 2 summarizes our recommendations.

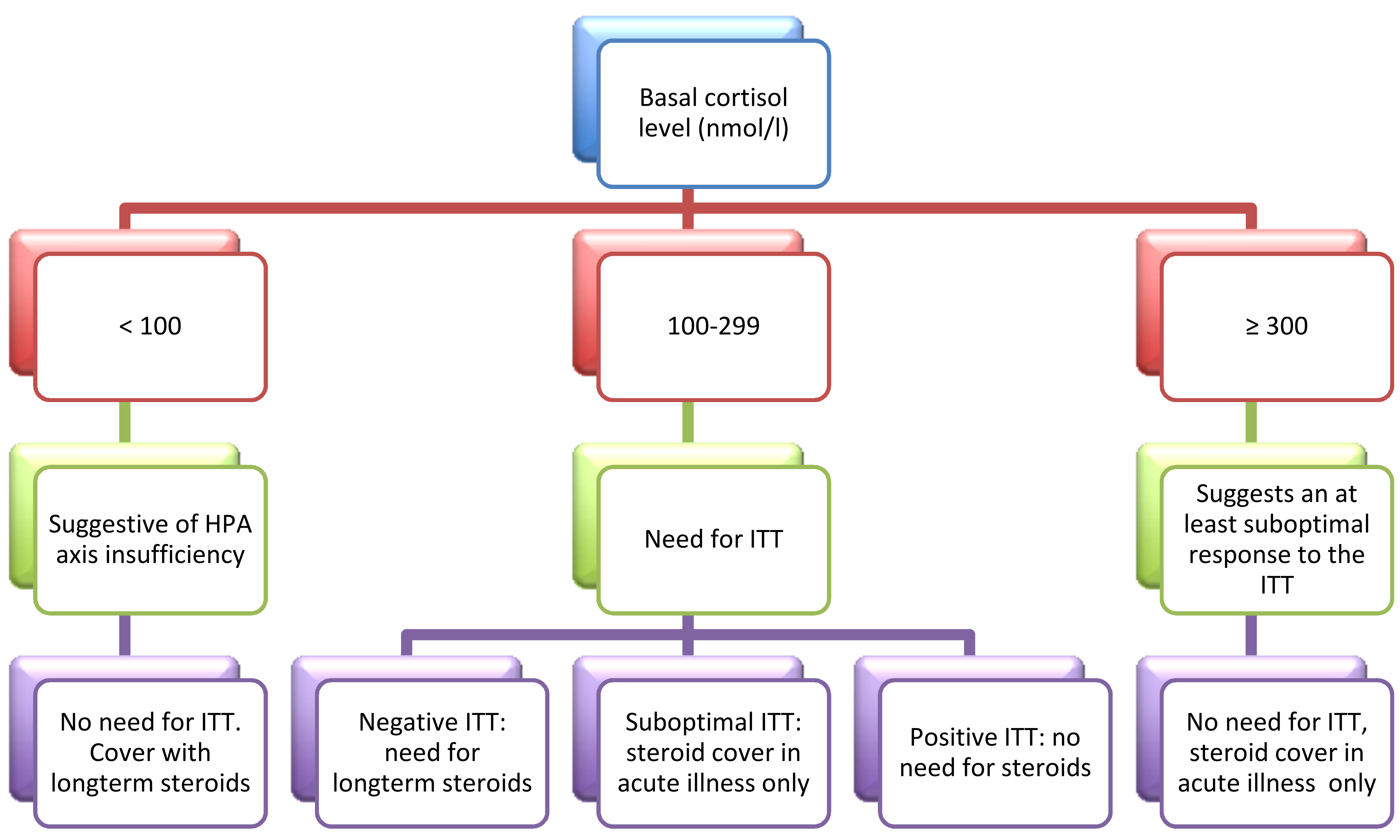

Figure 2: Algorithm with our recommendation about using the basal cortisol levels as a guide to decide upon the need for ITT and treatment options 